\title{
Clinical Features and Dynamics of T Cells-Related Markers in Immunocompetent Patients with Cytomegalovirus Hepatitis
}

\author{
Takuya Komura $\mathbb{D}^{1,}{ }^{1,2}$ Takashi Kagaya, ${ }^{1}$ Hideo Takayama, ${ }^{2}$ Masahiro Yanagi, ${ }^{2}$ \\ Takatoshi Yoshio, ${ }^{2}$ Saiho Sugimoto, ${ }^{2}$ Michiko Nishino, ${ }^{2}$ Noriaki Orita, ${ }^{2}$ Yoshiro Asahina, ${ }^{2}$ \\ Masashi Nishikawa, ${ }^{1,2}$ Shuichi Kaneko, ${ }^{2}$ and Masashi Unoura ${ }^{1}$ \\ ${ }^{1}$ Division of Gastroenterology NHO, Kanazawa Medical Center, Kanazawa, Japan \\ ${ }^{2}$ System Biology, Kanazawa University, Graduate School of Medical Science, Kanazawa, Japan \\ Correspondence should be addressed to Takuya Komura; t.komura0507@gmail.com
}

Received 8 May 2020; Revised 14 July 2020; Accepted 21 August 2020; Published 30 August 2020

Academic Editor: Alessandro Granito

Copyright (c) 2020 Takuya Komura et al. This is an open access article distributed under the Creative Commons Attribution License, which permits unrestricted use, distribution, and reproduction in any medium, provided the original work is properly cited.

\begin{abstract}
Aim. Cytomegalovirus (CMV) can cause hepatitis, encephalomyelitis, and pneumonitis in immunocompromised patients. In contrast, CMV infection of immunocompetent patients can lead to the development of infectious mononucleosis and is typically self-limiting; severe complications are rare. We evaluated the pathophysiology and immunological aspects of CMV hepatitis in recently immunocompetent adult patients. Methods. We examined the clinical features and outcomes of 47 adult immunocompetent patients with CMV hepatitis (29 men, 18 women; mean age, $34 \pm 11$ years) from January 2005 to August 2019 treated in our hospital. We also assayed T-cell activation to evaluate the immune responses in these patients. Results. Fever (74.5\%), hepatosplenomegaly $(74.5 \%)$, sore throat $(36.2 \%)$, headache $(31.9 \%)$, abdominal pain $(27.7 \%)$, lymphadenopathy $(23.4 \%)$, and skin rash (6.4\%) were present at admission. Complications included gastrointestinal injury (25.5\%), neuropathy (4.3\%), thrombocytopenia $(2.1 \%)$, and splenic infarction (2.1\%). All patients had a good clinical course without liver failure or transition to chronic liver injury. The time to recover from liver injury ranged from 12 to 142 days (mean, $43.4 \pm 28.7$ days). The serum sIL2R level, which reflects T-cell activation, was transiently elevated and correlated with the extent of hepatic inflammation. Conclusions. CMV hepatitis in immunocompetent individuals has a satisfactory outcome, but occasionally results in complications in other organs. The sIL-2R level has potential as a surrogate marker of hepatic inflammation in immunocompetent patients with CMV hepatitis.
\end{abstract}

\section{Introduction}

Reactivation of latent cytomegalovirus (CMV) infection or acquisition of primary CMV infection can result in encephalitis, pneumonitis, hepatitis, uveitis, retinitis, colitis, and graft rejection in immunocompromised patients [1]. In contrast, in immunocompetent patients, primary CMV infection typically manifests as an undifferentiated viral syndrome or an infectious mononucleosis-like syndrome. Therefore, CMV infection in such individuals is considered to be of minimal importance [2].

The seroprevalence of CMV in immunocompetent young adults worldwide has decreased to approximately $60 \%$ from $70-100 \%$ [3]. Therefore, the rate of primary CMV infection has increased recently. The disease is considered to be benign and self-limiting in immunocompetent individuals; however, some recent reports have indicated that CMV infection of immunocompetent patients can result in severe clinical manifestations [4]. Thus, further research is needed regarding CMV infection in recently immunocompetent individuals.

However, CMV infection induces large numbers of $\mathrm{CD} 8^{+} \mathrm{T}$ cells that retain effector functions and are dependent on $\mathrm{CD}^{+} \mathrm{T}$ cells help and costimulatory signals that home to peripheral organs [5]. The level of the soluble interleukin-2 receptor (sIL-2R) in peripheral blood reflects the extent of 
T-cell activation [6], which is correlated with the development of malignant lymphoma [7], rheumatoid arthritis [8], and IgG4-related disease [9]. However, the immune response in immunocompetent patients with CMV hepatitis is unclear.

In the present study, we examined the clinical features and outcomes of CMV-infected immunocompetent patients and assessed the immunological response to CMV infection by assaying the sIL-2R level and CD4/CD8 T-cells ratio.

\section{Materials and Methods}

The study was performed in accordance with the ethical guidelines of the 2013 Declaration of Helsinki; the study protocol was approved by the Institutional Review Board of the NHO Kanazawa Medical Center.

2.1. Patients. This study included 47 immunocompetent patients with CMV hepatitis (29 men, 18 women; mean age, $34 \pm 11$ years) who were diagnosed and treated at NHO Kanazawa Medical Center from January 2005 to August 2019 (Table 1). Immunocompetence was defined as the absence of current or prior malignant disease, steroid or immunomodulatory treatment, or immune disorders. All patients were positive for anti-CMV IgM in serum or for CMV antigenemia. Patients with liver injuries due to other etiologies were excluded. The serum sIL-2R level was assessed in 24 patients with CMV infection (17 men, 7 women; mean age, $61 \pm 7$ years) (Table 2). There were no differences in any measured parameter between males and females.

2.2. Clinical Features. At admission, we evaluated the following factors: white blood cell (WBC) count, frequency of lymphocytes, ratio of atypical lymphocytes, maximum C-reactive protein (CRP) level, maximum aspartate aminotransferase level (AST), maximum alanine aminotransferase (ALT) level, maximum total bilirubin (T-bil) level, minimum albumin (Alb) level, maximum prothrombin time- (PT-) international normalized ratio (INR), injuries to other organs, treatments, transition to hepatic failure, transition to chronic liver injury, interval to amelioration of hepatitis, and clinical outcome. Amelioration of hepatitis was defined as normalization of the serum ALT level.

\subsection{Immunological Parameters}

2.3.1. T-Cell Activation. In patients with CMV infection, $\mathrm{CD}^{+}{ }^{+} \mathrm{T}$-cells responses are dependent on $\mathrm{CD} 4^{+} \mathrm{T}$-cells aid and costimulatory signals. We assayed serum sIL-2R levels using the STACIA CLEIA kit (LSI Medience Corporation, Tokyo, Japan; normal range: $121-613 \mathrm{U} / \mathrm{mL}$ ) in 24 patients at admission and its correlations with the following factors: WBC count, lymphocyte count, CRP level, AST level, ALT level, T-bil level, minimum Alb level, and maximum PT-INR level. Finally, we examined the serum sIL-2R level after amelioration of hepatitis.
TABLE 1: Characteristics of the study subjects.

\begin{tabular}{lc}
\hline Characteristics & \\
\hline Gender, male/female & $29 / 18$ \\
Age & $34.0 \pm 10.9(20-58)$ \\
White blood cell counts & $9800 \pm 4000 / \mathrm{mm}^{3}$ \\
Lymphocyte ratio & $43.0 \pm 12.0 \%$ \\
Atypical lymphocyte ratio & $28(53.6 \%)$ \\
Maximum AST & $239 \pm 172 \mathrm{IU} / \mathrm{L}$ \\
Maximum ALT & $340 \pm 231 \mathrm{IU} / \mathrm{L}$ \\
Maximum CRP & $1.5 \pm 1.6 \mathrm{mg} / \mathrm{dL}$ \\
Maximum T-bil & $0.84 \pm 0.66 \mathrm{mg} / \mathrm{dL}$ \\
Minimal Alb & $4.1 \pm 0.40 \mathrm{~g} / \mathrm{dL}$ \\
Maximum PT-INR & $1.00 \pm 0.094$ \\
\hline
\end{tabular}

Data are expressed as means $\pm \mathrm{SD}$.

TABLE 2: Characteristics of the sIL-2R study subjects.

\begin{tabular}{lc}
\hline Characteristics & \\
\hline Gender, male/female & $17 / 7$ \\
Age & $35.3 \pm 10.3(21-58)$ \\
White blood cell counts & $9033 \pm 2751 / \mathrm{mm}^{3}$ \\
Lymphocyte ratio & $45.8 \pm 10.4 \%$ \\
Atypical lymphocyte ratio & $7.7(31 \%)$ \\
Maximum AST & $270 \pm 177 \mathrm{IU} / \mathrm{L}$ \\
Maximum ALT & $364 \pm 258 \mathrm{IU} / \mathrm{L}$ \\
Maximum CRP & $1.5 \pm 1.3 \mathrm{mg} / \mathrm{dL}$ \\
Maximum T-bil & $0.94 \pm 0.77 \mathrm{mg} / \mathrm{dL}$ \\
Minimal Alb & $4.0 \pm 0.4 \mathrm{~g} / \mathrm{dL}$ \\
Maximum PT-INR & $0.98 \pm 0.097$ \\
\hline
\end{tabular}

Data are expressed as means \pm SD.

2.3.2. Other Markers of Infection. We assayed the serum human immunodeficiency virus (HIV) antibody level in 15 patients. We also evaluated the frequencies of $\mathrm{CD}^{+}$and $\mathrm{CD}^{+} \mathrm{T}$ cells at admission and after amelioration of hepatitis in six patients. Six patients who provided written informed consent for evaluation of their $\mathrm{CD}^{+}$and $\mathrm{CD} 8^{+} \mathrm{T}$-cells numbers were enrolled. We measured the plasma HIV RNA level using the Roche TaqMan assay (lower limit of detection, 20 copies $/ \mathrm{mL}$ ); we assayed $\mathrm{CD}^{+}$and $\mathrm{CD}^{+}$T-cells subsets by flow cytometry. Serum sIL-2R levels and CD $4^{+}$ and $\mathrm{CD}^{+} \mathrm{T}$-cell counts are routinely measured in our hospital when needed. These parameters served as measures of T-cell activation.

2.4. Statistical Analysis. Statistical analysis was performed using Microsoft Excel software (Microsoft, Redmond, WA, USA). Data are shown as medians and interquartile ranges, as well as means and standard errors of the mean. Differences in variables before and after normalization of the ALT level were examined by paired Student's $t$-tests after a normal distribution had been confirmed. A value of $P<0.05$ was considered indicative of statistical significance. The Pearson correlation coefficient was calculated to assess correlations of the serum sIL-2R level with a variety of laboratory parameters. 


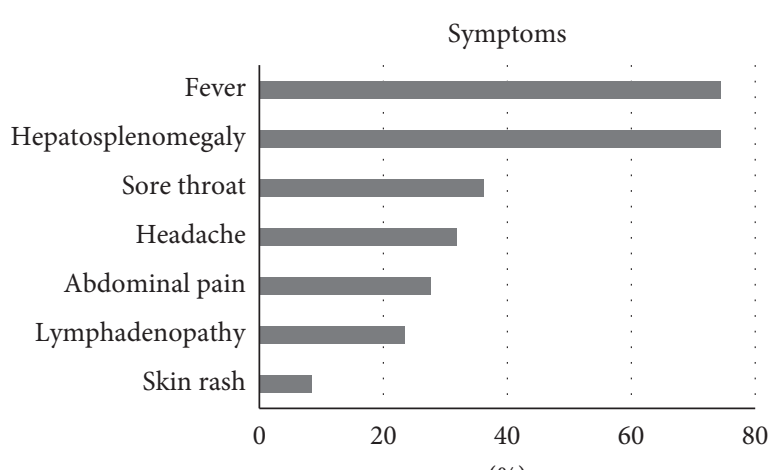

(\%)

(a)

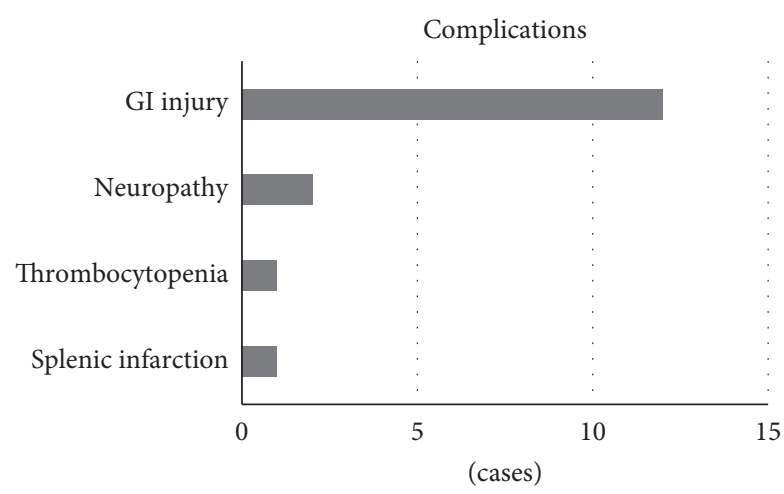

(b)

FIGURE 1: Some clinical symptoms and complications of CMV hepatitis in 47 immunocompetent individuals. (a) The frequency of some clinical symptoms is shown. Fever, 35 patients (74.5\%); hepatosplenomegaly, 35 patients (74.5\%); sore throat, 17 patients (36.2\%); headache, 15 patients (31.9\%); abdominal pain, 13 patients (27.7\%); lymphadenopathy, 11 patients (23.4\%); and skin rash, 4 patients (6.4\%) on arrival to our hospital, respectively. (b) The frequency of some clinical complications is shown. GI injury, 7 patients (6.4\%); neuropathy, 2 patients (6.4\%); thrombocytopenia, 1 patient (6.4\%); and splenic infarction, 1 patient (6.4\%), respectively.

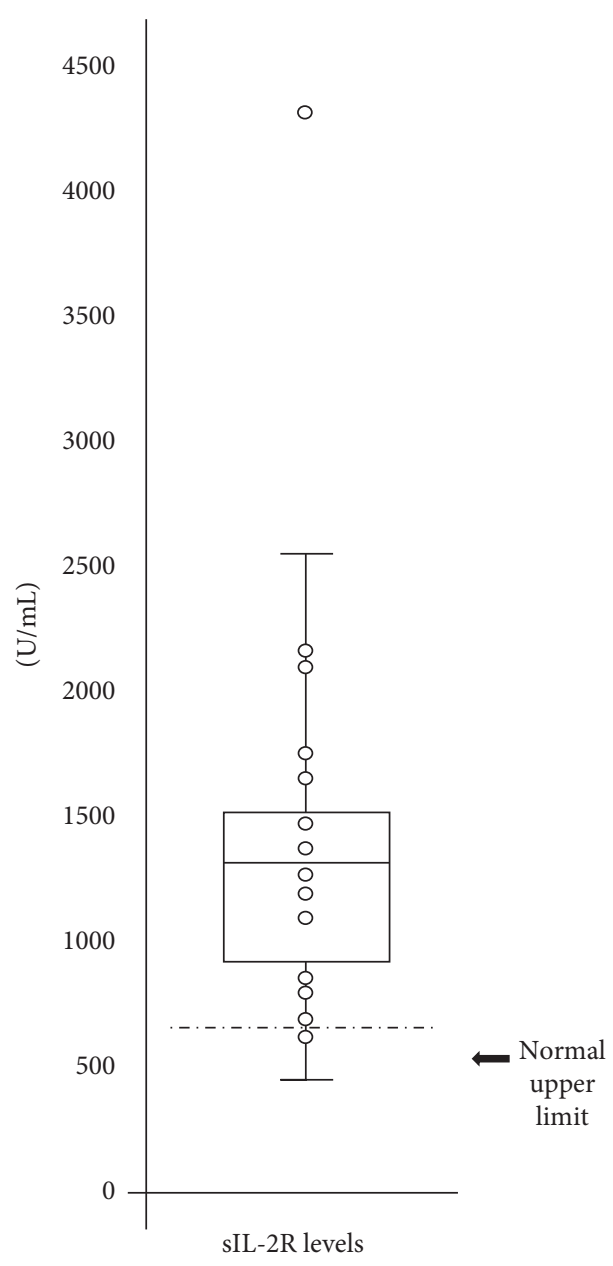

(a)

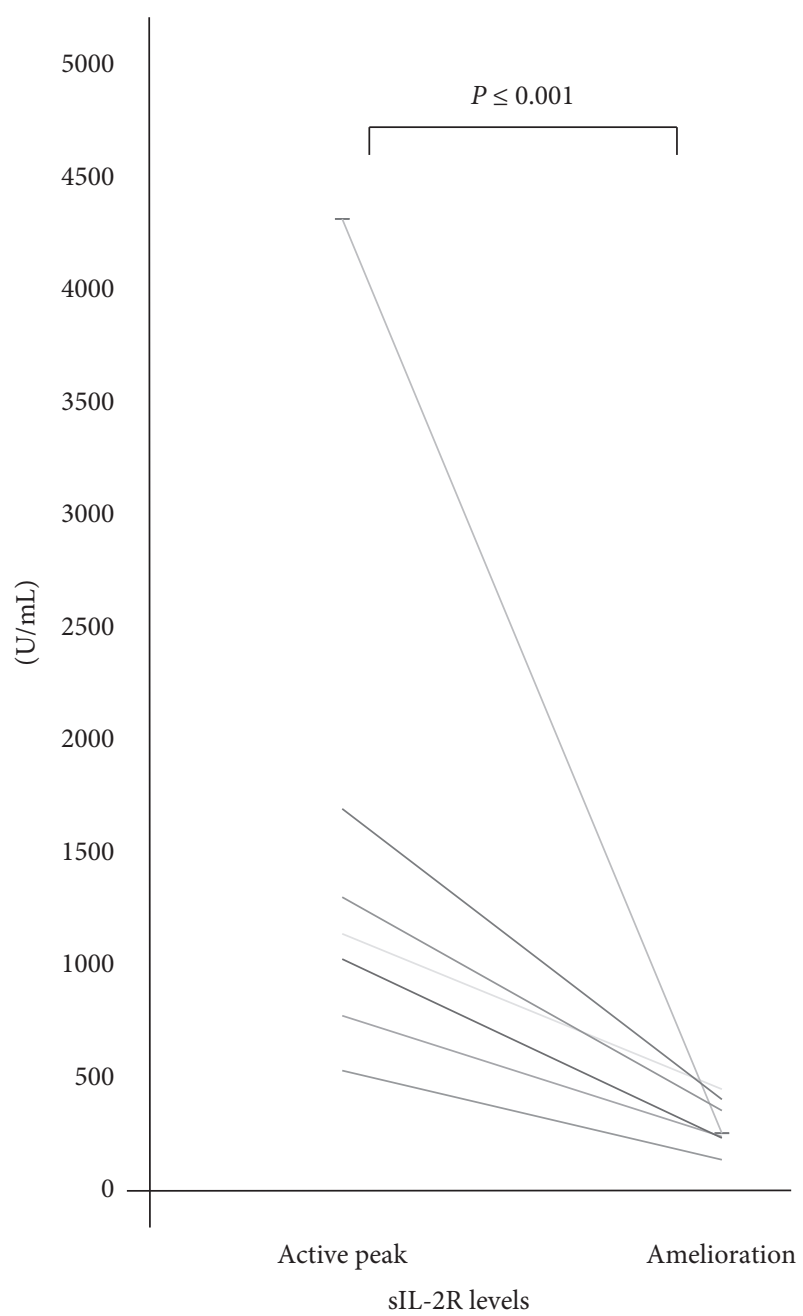

(b)

FIGURE 2: sIL-2R levels in serum of 24 patients with CMV hepatitis. (a) sIL-2R levels in serum of patients with CMV hepatitis were elevated on arrival. Dot indicated each sIL-2R levels of CMV patients. Data are expressed as means \pm SEM. (b) 7 patients could be measured at sIL-2R levels after normalization of ALT levels which suggested calm down of hepatitis. These levels were significantly decreased. ${ }^{*} P<0.05$. 


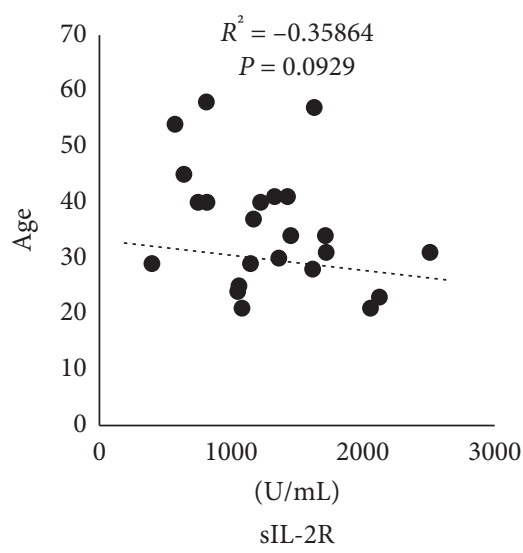

(a)

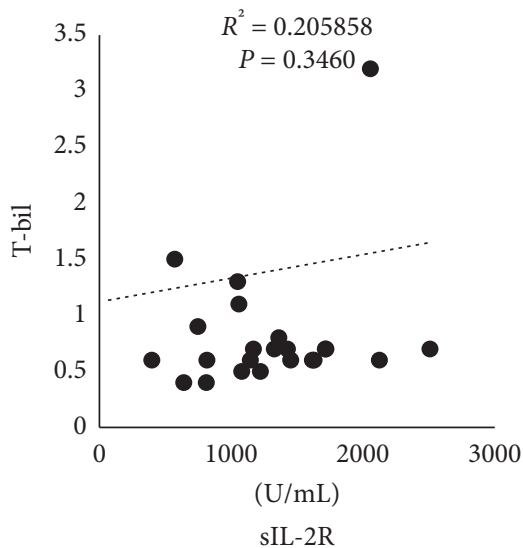

(d)

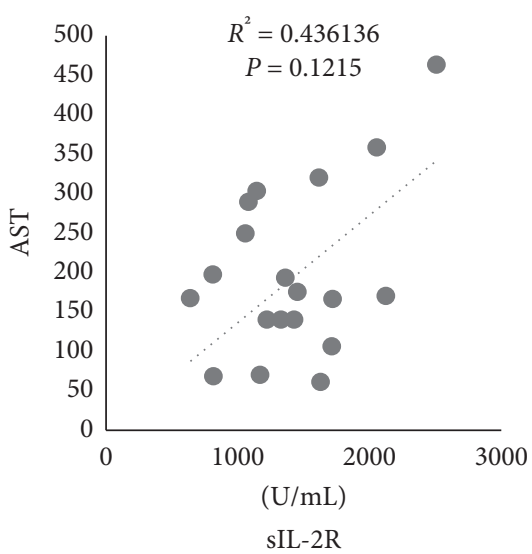

(g)

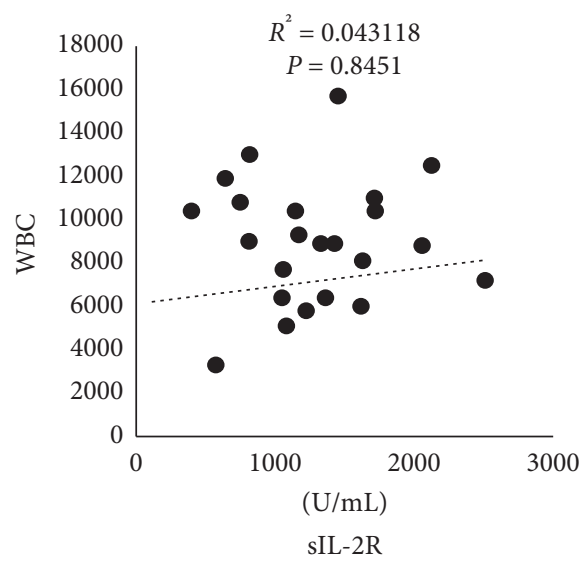

(b)

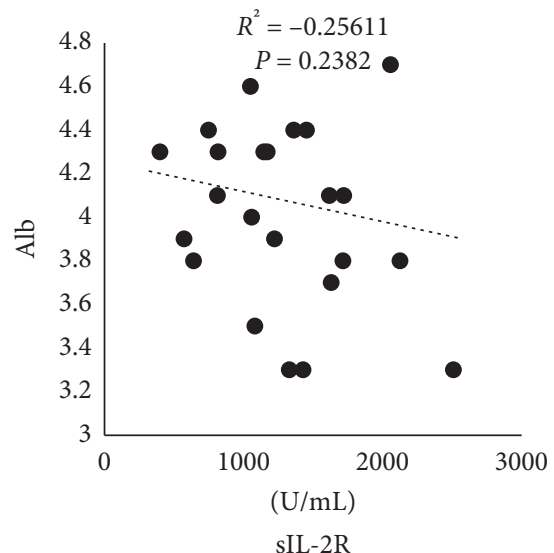

(e)

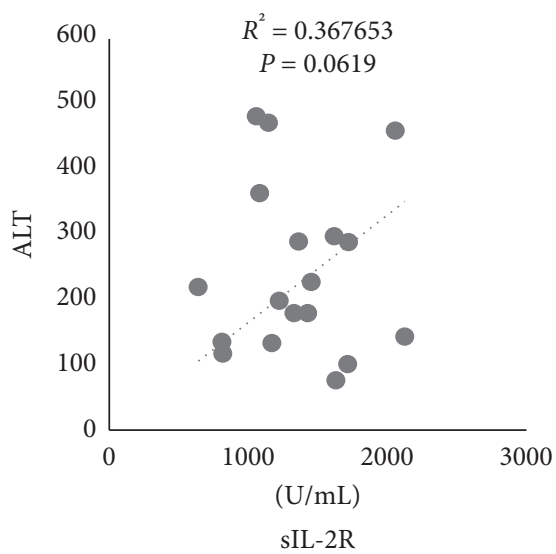

(h)

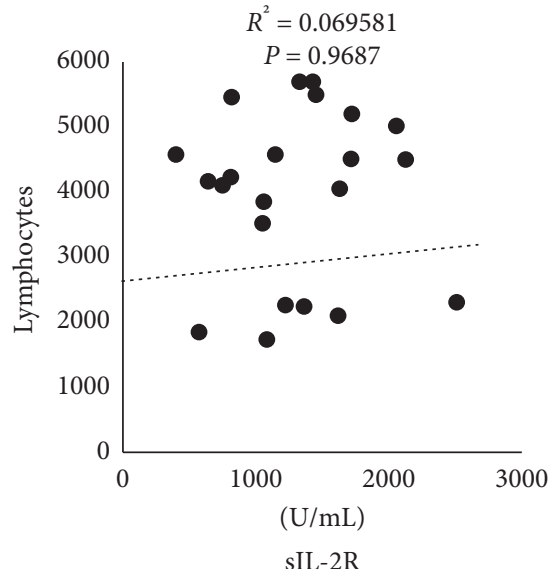

(c)

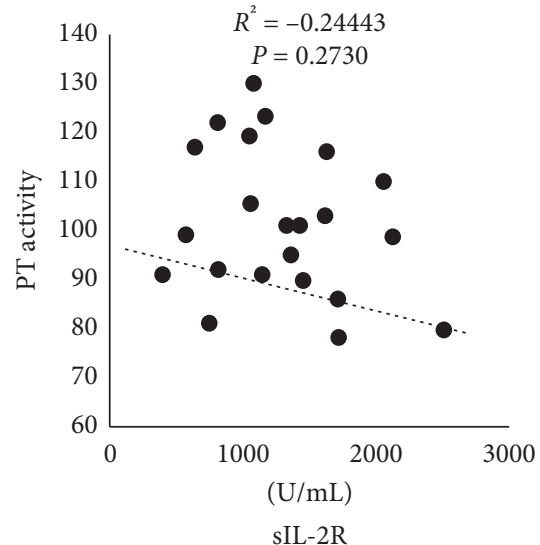

(f)

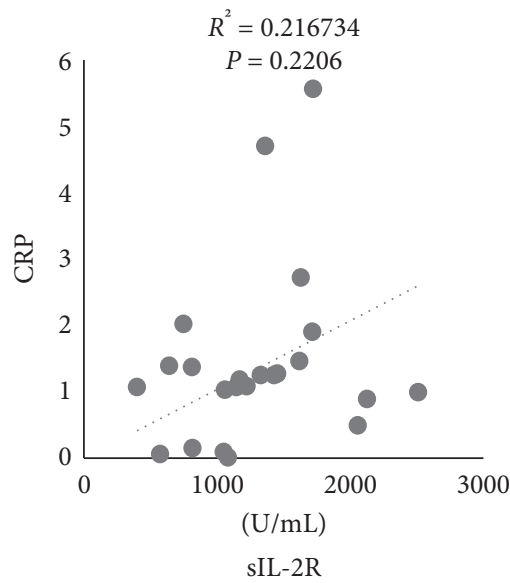

(i)

FIgURE 3: Correlation with sIL-2R levels and some laboratory data in peripheral blood of 24 patients with CMV hepatitis. (a) Age (years), (b) WBC counts $\left(/ \mathrm{mm}^{3}\right),(\mathrm{c})$ lymphocytes counts $\left(/ \mathrm{mm}^{3}\right)$, (d) T-bil (mg/dL), (e) Alb (g/dL), (f) PT activity (\%), (g) AST (IU/L), (h) ALT (IU/L), and (i) CRP $(\mathrm{mg} / \mathrm{dL})$, respectively. (d-f) Hepatitis reserve ability.

\section{Results}

3.1. Demographics. We retroactively assessed 47 immunocompetent patients with CMV hepatitis. The demographic characteristics of the patients are shown in Table 1. The serum AST and ALT levels were moderately elevated, and the CRP level was slightly elevated. The T-bil, Alb, and PTINR levels were not decreased, suggesting that the hepatic reserve was not impaired. Fever, hepatosplenomegaly, sore throat, headache, abdominal pain, lymphadenopathy, and skin rash at admission were found in 35 (74.5\%), 35 (74.5\%), 17 (36.2\%), 15 (31.9\%), $13(27.7 \%), 11(23.4 \%)$, and $4(6.4 \%)$ 


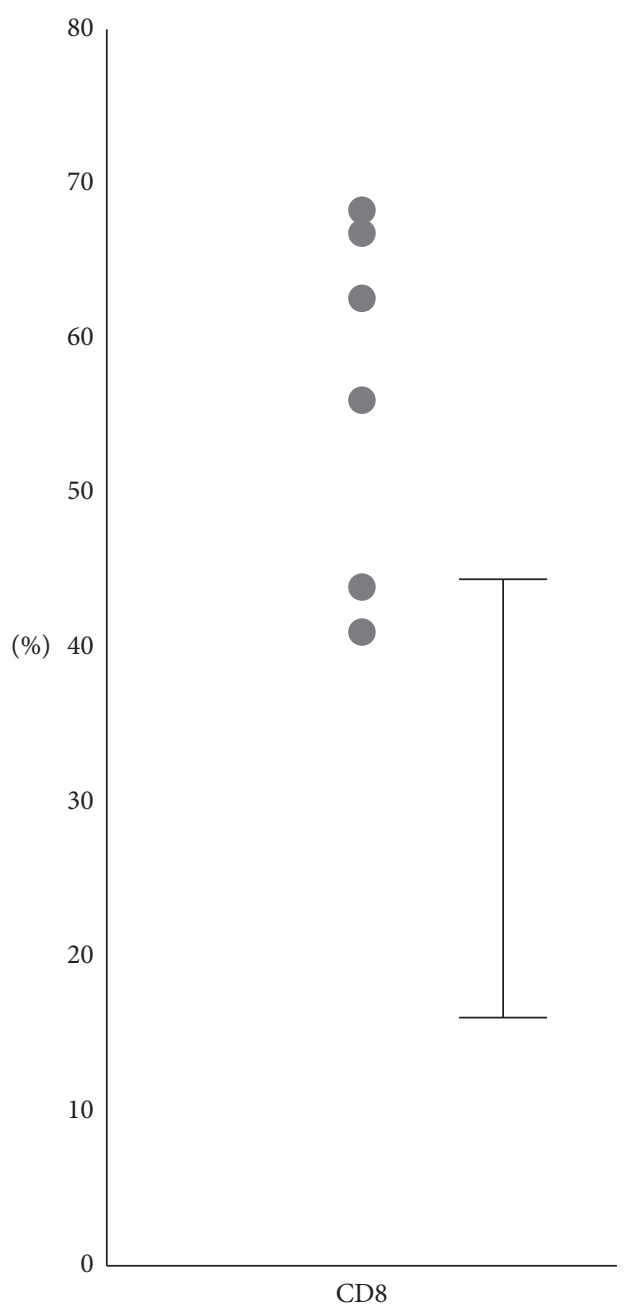

(a)

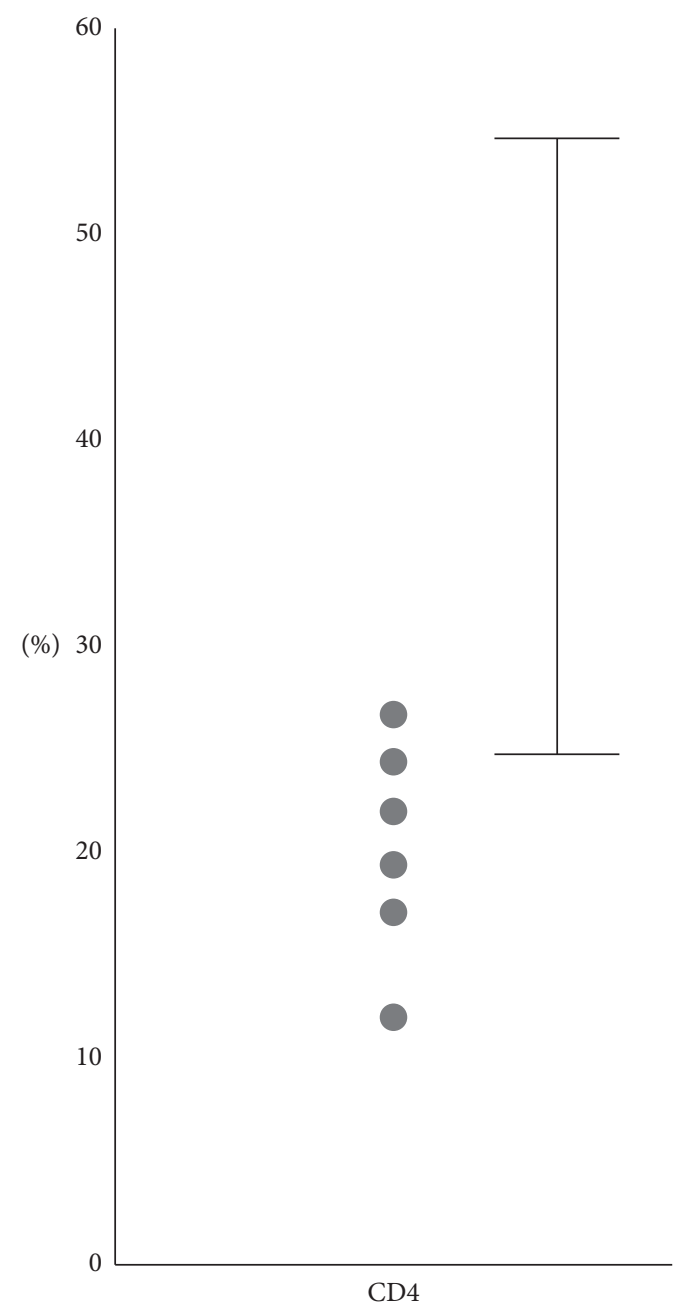

(b)

FIGURE 4: The CD4/CD8 ratio in the peripheral blood of six patients with CMV hepatitis, measured at the time of admission. (a) The CD8 ${ }^{+}$ $\mathrm{T}$-cell proportions were high. (b) The $\mathrm{CD} 4^{+} \mathrm{T}$-cell proportions were low. The solid lines indicate the normal ranges of the $\mathrm{CD} 4^{+}$and $\mathrm{CD} 8^{+} \mathrm{T}$ cell proportions.

patients, respectively (Figure 1(a)). Gastrointestinal (GI) injury occurred in 12 patients (25.5\%), neuropathy in two patients $(4.3 \%)$, thrombocytopenia in one patient $(2.1 \%)$, and splenic infarction in one patient (2.1\%) (Figure 1(b)).

3.2. Outcomes. All patients survived; one was treated with ganciclovir (an anti-CMV agent) for 10 days because of a high fever and severe general malaise caused by bacteremia. No patient developed hepatic failure or chronic liver injury. In addition, no patients showed a worsened serum PT-INR or Alb level. The serum T-bil levels in two patients were 3.5 and $3.2 \mathrm{mg} / \mathrm{dL}$, and thus slightly elevated. The ALT levels of all patients normalized after $43.4 \pm 28.7$ days from the peak.

We observed several complications associated with CMV infection in the absence of hepatitis. Gastrointestinal (GI) injury was classified as gastric ulcer and erosion (seven patients), duodenal ulcer and erosion (two patients), esophageal ulcer (one patient), and colitis (two patients); the diagnoses were pathologically proven by endoscopically obtained biopsy. Antacids were given to 10 patients. The other symptoms were treated palliatively. Two patients became neurological diseases. One patient was diagnosed with Guillain-Barre syndrome and was prescribed an oral steroid and $\gamma$-globulin for 5 days. Another patient exhibited mild neuropathy, but the clinical course was good in the absence of treatment. One patient developed thrombocytopenia, and 10 units of platelet concentrate were infused; the clinical course was good. The patient with splenic infarction did not require any treatment and exhibited a good clinical course.

3.3. Immunological Parameters. The characteristics of the study patients are shown in Table 2. The serum sIL-2R level was significantly elevated at the peak of hepatitis (range 399-4,287 U/mL; mean 1,414 U/mL; Figure 2(a)) but became normal after amelioration of hepatitis (Figure 2(b)). The sIL-2R level was correlated moderately with the AST, ALT, and CRP levels, which reflect liver inflammation and 
damage (Figure 3(a)), and was correlated weakly with the serum T-bil, Alb, and PT-INR levels (Figure 3(b)), which reflect the liver reserve. The serum sIL-2R level, which reflects $\mathrm{T}$-cell activation, was transiently elevated and correlated with disease activity.

No anti-HIV antibodies were detected. The frequency of $\mathrm{CD}^{+}$T cells was transiently elevated (Figure 4(a)), while the frequency of $\mathrm{CD}^{+} \mathrm{T}$ cells was reduced, during active hepatitis (Figure 4(b)). Following amelioration of hepatitis, the (CD4/CD8) ratio became normalized (data not shown).

\section{Discussion}

All immunocompetent patients with CMV hepatitis exhibited transient symptoms, although antivirals were typically not required. While most patients exhibited a benign clinical course, some developed complications that required palliative treatment.

It is often considered that CMV infection in immunocompetent individuals is self-limiting and does not require treatment [10]. However, we detected a variety of common complications of CMV infection, including GI injury, neuropathy, and thrombosis [11-13]. The GI injuries were mild and were treated successfully. Vascular thrombosis is a potential manifestation of CMV infection in immunocompetent individuals. A variety of veins can be affected by CMV infection, presumably due to the procoagulant activity of CMV $[14,15]$. The suppression of hematopoiesis associated with CMV infection is caused by direct inhibition of the growth of progenitor hematopoietic cells, resulting in immunological abnormalities and thrombocytopenia [16]. A potential reason for the relatively large number of complications observed in our study was that we chose patients with CMV-induced hepatitis, all of whom exhibited CMV infection-induced organ injury; thus, they likely had severe CMV infection. Hence, CMV infection in immunocompetent patients can occasionally trigger relatively severe complications and should be treated aggressively [11].

IL-2 is a multipotent cytokine that plays a crucial role in signaling pathways that control the differentiation and homeostasis of pro- and anti-inflammatory $\mathrm{T}$ cells by binding with high affinity to trimeric IL-2R. Hence, sIL-2R reflects the level of T-cell activation [17]. The sIL-2R level was correlated with the AST, ALT, CRP, T-bil, Alb, and PTINR levels, suggesting that the sIL-2R level reflects the extent of CMV hepatitis activity in infected patients, as in those with systemic lupus erythematosus and sarcoidosis, both of which feature T-cell activation [18, 19]. The transient decrease in the (CD4/CD8) ratio is in line with that of an earlier report suggesting that CMV infection increases the numbers of $\mathrm{CD}^{+} \mathrm{T}$ cells that exert effector functions [5].

In conclusion, patients with CMV hepatitis had satisfactory outcomes, such that few required antiviral agents. However, some complications required transient palliative treatment. The serum sIL-2R level, which reflects the T-cell response, was identified as a biomarker of disease activity in immunocompetent patients with CMV hepatitis.

\section{Data Availability}

The data used to support the findings of this study are included within the article.

\section{Ethical Approval}

This study was approved by the Institutional Review Board and was performed in accordance with the Declaration of Helsinki.

\section{Conflicts of Interest}

The authors declare that they have no conflicts of interest.

\section{References}

[1] V. C. Emery, "Cytomegalovirus: recent progress in understanding pathogenesis and control," QJM, vol. 105, no. 5, pp. 401-405, 2012.

[2] J. I. Cohen and G. R. Corey, "Cytomegalovirus infection in the normal host," Medicine, vol. 64, no. 2, pp. 100-114, 1985.

[3] M. J. Cannon, D. S. Schmid, and T. B. Hyde, "Review of cytomegalovirus seroprevalence and demographic characteristics associated with infection," Reviews in Medical Virology, vol. 20, no. 4, pp. 202-213, 2010.

[4] G. Carpani, S. Foresti, R. Dell'Oro, G. Grassi, and M. Bombelli, "Severe systemic cytomegalovirus infection in an immunocompetent patient outside the intensive care unit: a case report," BMC Infectious Diseases, vol. 19, no. 1, p. 34, 2019.

[5] P. Klenerman and A. Oxenius, "T cell responses to cytomegalovirus," Nature Reviews Immunology, vol. 16, no. 6, pp. 367-377, 2016.

[6] L. A. Rubin, C. C. Kurman, M. E Fritz et al., "Soluble interleukin 2 receptors are released from activated human lymphoid cells in vitro," Journal of Immunology (Baltimore, Md: 1950), vol. 135, no. 5, pp. 3172-3177, 1985.

[7] C. A. Lindqvist, L. H. Christiansson, B. Simonsson, G. Enblad, U. Olsson-Strömberg, and A. S. I. Loskog, "T regulatory cells control T-cell proliferation partly by the release of soluble CD25 in patients with B-cell malignancies," Immunology, vol. 131, no. 3, pp. 371-376, 2010.

[8] L. A. Rubin, K. M. Snow, C. C. Kurman, D. L Nelson, and E. C Keystone, "Serial levels of soluble interleukin 2 receptor in the peripheral blood of patients with rheumatoid arthritis: correlations with disease activity," The Journal of Rheumatology, vol. 17, no. 5, pp. 597-602, 1990.

[9] A. F. Karim, L. E. M. Eurelings, R. D. Bansie, P. M. Van Hagen, J. A. M. Van Laar, and W. A. Dik, "Soluble interleukin-2 receptor: a potential marker for monitoring disease activity in IgG4-related disease," Mediators of Inflammation, vol. 2018, Article ID 6103064, 6 pages, 2018.

[10] J. F. Faucher, B. Abraham, M. Segondy, O. Jonquet, J. Reynes, and F. Janbon, "Acquired cytomegalovirus infections in immunocompetent adults: 116 cases," Presse Medicale, vol. 27, pp. 1774-1779, 1998.

[11] P. I. Rafailidis, E. G. Mourtzoukou, I. C. Varbobitis, and M. E. Falagas, "Severe cytomegalovirus infection in apparently immunocompetent patients: a systematic review," Virology Journal, vol. 5, no. 1, p. 47, 2008.

[12] F. C. Devetag and L. Boscariolo, "Cytomegalovirus meningoencephalitis with paroxysmal course in immunocompetent 
adults: a new nosographical entity," European Neurology, vol. 44, no. 4, pp. 242-247, 2000.

[13] M. Eddleston, S. Peacock, M. Juniper, and D. A. Warrell, "Severe cytomegalovirus infection in immunocompetent patients," Clinical Infectious Diseases, vol. 24, no. 1, pp. 52-56, 1997.

[14] P. Warrell, V. Delbos, J. M. Chennebault, C. Payan, and E. Pichard, "Vascular thrombosis and acute cytomegalovirus infection in immunocompetent patients: report of 2 cases and literature review," Clinical Infectious Diseases, vol. 36, pp. 134-139, 2003.

[15] A. Squizzato, W. Ageno, A. Cattaneo et al., "A case report and literature review of portal vein thrombosis associated with cytomegalovirus infection in immunocompetent patients," Clinical Infectious Diseases, vol. 44, no. 2, pp. 13-16, 2020.

[16] K. Nomura, Y. Matsumoto, Y. Kotoura et al., "Thrombocytopenia due to cytomegalovirus infection in an immunocompetent adult," Hematology, vol. 10, no. 5, pp. 405-406, 2005.

[17] S. H. Ross and D. A. Cantrell, "Signaling and function of interleukin-2 in T lymphocytes," Annual Review of Immunology, vol. 36, no. 1, pp. 411-433, 2018.

[18] E. J. Ter Borg, G. Horst, P. C. Limburg, and C. G. M. Kallenberg, "Changes in plasma levels of interleukin2 receptor in relation to disease exacerbations and levels of anti-dsDNA and complement in systemic lupus erythematosus," Clinical \& Experimental Immunology, vol. 82, pp. 21-26, 1990.

[19] A. D. M. Vorselaars, C. H. M. Van Moorsel, P. Zanen et al., "ACE and sIL-2R correlate with lung function improvement in sarcoidosis during methotrexate therapy," Respiratory Medicine, vol. 109, no. 2, pp. 279-285, 2015. 